\title{
Kernos
}

Revue internationale et pluridisciplinaire de religion grecque antique

15 | 2002

Varia

\section{Maria Luisa NAVA, Massimo OSANNA (éds), Rituali per una Dea Lucana. Il santuario di Torre di Satriano}

\section{Sandrine Ducaté-Paarmann}

\section{OpenEdition \\ Journals}

\section{Édition électronique}

URL : http://journals.openedition.org/kernos/1450

DOI : $10.4000 /$ kernos. 1450

ISSN : 2034-7871

\section{Éditeur}

Centre international d'étude de la religion grecque antique

Édition imprimée

Date de publication : 1 janvier 2002

ISSN : 0776-3824

\section{Référence électronique}

Sandrine Ducaté-Paarmann, « Maria Luisa nava, Massimo osanna (éds), Rituali per una Dea Lucana. II santuario di Torre di Satriano », Kernos [En ligne], 15 | 2002, mis en ligne le 22 avril 2011, consulté le 24 septembre 2020. URL : http://journals.openedition.org/kernos/1450 ; DOI : https://doi.org/10.4000/ kernos. 1450 
difficultés. La principale étant que le grand chapiteau hathorique publié dès 1985 par A. Hermary, qu'il estime appartenir à cet hypothétique sanctuaire, provient d'une zone qui a depuis longtemps été identifiée comme appartenant au palais lui-même ( $B C H, 107,1983$, p. 967, fig. 14). Ensuite l'A. dénie tout caractère votif à certains types que l'on rencontre dans la petite plastique qui fut trouvée dans le remblai couvrant les magasins du palais; pour lui, ils constituent une « partie du mobilier de la vie quotidienne [qui] rappelait les principales activités des maîtres du lieu ». Pareille hypothèse ne laisse pas d'étonner : tout d'abord, ces nombreux objets ne peuvent provenir que de sanctuaires que l'on peut imaginer, à l'exemple de Vouni, sis tout autour du palais, voire dans le corps de l'édifice lui-même. Deux de ces ensembles cultuels palatiaux sont d'ailleurs présentés dans l'introduction de l'ouvrage (p. 5-6). Ensuite, l'interprétation de l'A. peut à la rigueur expliquer la fonction de ces artefacts, mais laisse dans l'ombre leur nature. Sauf à imaginer ces « maîtres des lieux » comme dotés d'une complexion particulièrement ludique, qu'en faire d'autre sinon des paraphernalia et des ex-voto de cultes palatiaux?

Dans un chapitre conclusif très convaincant (p. 167-169), l'A. confronte les enseignements que l'on peut tirer de ce matériel pour le culte d'Aphrodite au sanctuaire sommital avec les textes littéraires et les nombreuses études qu'il a luimême déjà consacrées au sujet.

Thierry Petit

(Université de Strasbourg II)

Maria Luisa Nava, Massimo Osanna (éds), Rituali per una Dea Lucana. Il santuario di Torre di Satriano, Università degli studi della Basilicata, Soprintendenza Archeologica della Basilicata, 2001. 1 vol, $22 \times 28 \mathrm{~cm}, 150$ p., nbr. ill. (catalogue d'exposition).

Le site de Torre di Satriano situé au sud-ouest de Potenza (Basilicate) a fait l'objet, en 2000, de nouveaux sondages archéologiques entrepris conjointement par la surintendance (M.L. Nava) et l'Université de Basilicate (sous la direction de M. Osanna). Les fouilles qui ont porté sur le sanctuaire lucanien découvert en 1987/88 par l'équipe de E. Greco ( $c f$. Satriano 1987/88. Un biennio di ricerche archeologiche, Naples, 1988) au sud-ouest de la zone urbaine complètent ainsi les données topographiques du site rassemblées une première fois en 1970 par R.R. Holloway ( $c f$. Satrianum. The Archaeological Investigations Conducted by Brown University in 1966 and 1967, Providence, 1970). Ce sont les résultats de ces nouvelles investigations qui sont publiées dans le présent ouvrage réalisé à l'occasion de l'exposition Rituali per una dea lucana organisée au musée provincial de Potenza et faisant suite à deux autres manifestations intitulées L'arcbeologia in Basilicata et Archeologia dell'acqua in Basilicata. Ce catalogue est composé de neuf chapitres rédigés par une quinzaine d'auteurs et parcourant les différentes phases de fréquentation du site depuis le Bronze Moyen jusqu'au Moyen Âge, en accordant toutefois une plus grande importance à la période lucanienne ( $\mathrm{V}^{\mathrm{e}}-\mathrm{III} \mathrm{e}^{\mathrm{e}} \mathrm{s} . \mathrm{av} . \mathrm{J} . \mathrm{C}$.). Dans le premier chapitre (Un territorio dell'Ttalia antica par M. Osanna et A. Russo, p. 13-16) les A. présentent les cadres géographiques et humains de la Lucanie antique (Basilicate et partie méridionale de la Campanie actuelle). Le chapitre suivant (Prima del Lucani par M. Osanna et M.M. Sica, p. 1728) est consacré à la description du site de Torre di Satriano et de son environnement géographique (fleuves, sources, etc.) ainsi qu'aux populations qui ont occupé l'endroit depuis le Bronze Moyen jusqu'au ves. av. J.-C. Dans le troisième chapitre (I Lucani par M. Osanna, p. 29-32), l'A. met en évidence les différents changements que l'archéologie permet d'observer dans cette région entre le $\mathrm{v}^{\mathrm{e}}$ et 
le $\mathbf{I V}^{\mathrm{e}} \mathrm{s}$. av. J.-C. (réorganisation du territoire, mise en place de fortifications, apparition de nouveaux sanctuaires) et liés à l'arrivée d'une population nouvelle, appelée lucanienne. On regrettera ici l'absence d'une synthèse sur l'origine de ce peuple et les circonstances de son installation en Basilicate. D'où viennent les Lucaniens et qui sont-ils? Voilà deux questions qui auraient bien mérité d'être développées dans un chapitre intitulé "Les Lucaniens". Dans les deux chapitres suivants sont exposés les résultats de la dernière campagne de fouilles menée au sanctuaire. Ainsi, le chapitre IV (Il santuario di Torre di Satriano par A. d'Alessio, M. Osanna et M.M. Sica, p. 33-44) présente les structures des deux édifices découerts par E. Greco et interprétés comme un sacellum et une "salle de banquet " flanquée d'une "cuisine " ainsi que les deux fosses (A et $B$ ) dégagées lors de la campagne de 2000 et situées à l'est de ces deux constructions. Dans le chapitre V (I materiali del santuario, p. 45-106) sont décrits les objets retrouvés dans ces fosses et présentés d'après le classement suivant : Ex-voto de terre cuite, dont certaines figurines montrent une nette influence paestane (par I. Battiloro, M. Blasi et C. Guardascione), Thymiateria (par M. Di Lieto), Céramique (par M. Blasi, L. Colangelo, R. Nigro, Cl. Pouzadoux et T. Virtuoso) et Métaux (par A. Bruscella, A. Di Noia et C. Guardascione). Le chapitre VI (Azioni rituali e offerte votive par M. Osanna et T. Giammatteo, p. 107-122) est consacré au thème même de l'exposition à savoir les actions rituelles telles que le sacrifice du chien souvent lié aux divinités protectrices des accouchements, le banquet rituel et la libation. Ces différentes pratiques connues aussi bien chez les Grecs que chez les Italiques sont attestées à Torre di Satriano par la découverte d'ossements canins scellant une déposition d'offrandes, d'ustensiles de cuisine et de services de table ainsi que d'un pilier dans lequel était insérée une phiale à libation. Ce chapitre se termine par des observations générales sur les contextes de découvertes des offrandes et la terminologie utilisée par les archéologues pour définir des ensem-

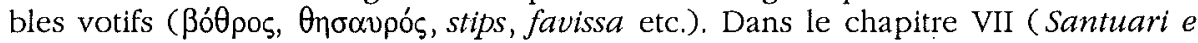
culti dei Lucani par C. Masseria et M.C. D'Anisi, p. 123-134), les A. passent en revue les principaux lieux cultuels attestés en Basilicate depuis la fin du $\mathrm{vI}^{\mathrm{e}}-\mathrm{v}^{\mathrm{e}} \mathrm{S}$. jusqu'à l'arrivée des Romains et s'attachent plus spécialement au célèbre sanctuaire de Rossano di Vaglio ainsi qu'à sa divinité tutélaire, Méfitis. Ce sous-chapitre relatif à la déesse osco-ombrienne expose les diverses étymologies proposées pour son nom, la diffusion et les caractéristiques de son culte. On s'étonnera de ne pas y trouver mentionné l'ouvrage incontournable de M. Lejeune (Méfitis d'après les dédicaces lucaniennes de Rossano di Vaglio, Louvain-La-Neuve, 1990). Enfin, les chapitres VIII (L'età romana par B. Serio, p. 135-138) et IX (La città medioevale: Satrianum par A. Ricci, p. 139-143) sont respectivement consacrés à la romanisation de la Lucanie et l'abandon du sanctuaire de Torre du Satriano ainsi qu'à la renaissance de la ville au Moyen Âge sous le nom de Satrianum. Ce catalogue qui sert en quelque sorte de rapport préliminaire de la fouille 2000 a le mérite de faire connaître rapidement au public les résultats provisoires des nouvelles investigations menées au sanctuaire lucanien. C'est là un point positif dont peu de fouilleurs peuvent se vanter! Il ne s'agit pas d'un simple catalogue d'objets agrémenté de quelques plans archéologiques mais d'un petit ouvrage de synthèse intégrant non seulement la publication des nouvelles trouvailles mais aussi l'état des connaissances actuelles sur les autres sanctuaires lucaniens en mettant clairement en évidence les affinités et les différences. Une grande attention est également apportée au paysage et la topographie de cette région baignée par de nombreux fleuves et sources qui ont joué un rôle important dans la localisation de ces lieux sacrés et la pratique des activités cultuelles. Cet ouvrage vient ainsi compléter le petit catalogue de l'exposition tenue à Rome en 1998 et intitulée "Il sacro e l'acqua. Culti indigeni in Basilicata", Le sous-chapitre 
traitant des sacrifices de chiens est particulièrement intéressant pour l'étude des rituels lucaniens relatifs aux "dépôts d'offrandes" scellés par des ossements canins brûlés. Le parallélisme avec l'un des botbroi de l'Héraion du Sele ouvre par ailleurs des perspectives de recherches intéressantes sur les actes rituels et leurs circonstances ainsi que sur le choix des animaux sacrifiés en milieu lucanien. On déplorera toutefois la médiocrité de la qualité des clichés couleur et noir et blanc (impression complètement floue), le manque de clarté des cartes et l'absence de certains plans. Ainsi, la carte de la Lucanie présentée au chapitre I est non seulement trouble mais la taille du caractère typographique choisi pour les noms des villes rend toute lecture impossible. Il en est de même pour les noms et la légende de la carte de la figure 5 au chapitre II relative à la distribution des nécropoles archaïques de la région de Torre di Satriano. On aurait également aimé retrouver un plan général du site (éventuellement repris de l'ouvrage de R.R. Holloway, ill. 2 et complété par les données récentes) qui aurait aidé le lecteur à localiser plus facilement le sanctuaire par rapport au secteur d'habitat et des nécropoles (les seuls plans du lieu de culte étant ceux des secteurs de fouilles présentés en dehors de tout contexte topographique). Espérons donc que le rapport de fouille définitif s'accompagne de nombreuses cartes utiles et lisibles ainsi que de photographies de qualité. Une autre remarque porte sur le manque de soin apporté aux références et à la bibliographie. À plusieurs reprises des références citées dans un texte n'ont pas été reprises dans la bibliographie générale en fin de volume (par ex. Lejeune 1967, 1971 et 1972; Lavagnini 1923; Lepore 1972 ou encore Prosdocimi 1988). En dehors de ces quelques reproches, Rituali per una dea lucana reste un ouvrage important et indispensable pour qui s'intéresse aux cultes lucaniens, que ce soit pour la topographie des sanctuaires, l'architecture des édifices sacrés ou encore les activités rituelles, qu'il s'agisse des banquets ou de l'ensevelissement des offrandes votives.

Sandrine Ducaté-Paarmann (Université de Paris IV-Sorbonne)

Silvia Lanzi, Theos Anaitios. Storia della teodicea da Omero ad Agostino, présentation de G. Casadio, Roma, Il Calamo, 2000. 1 vol. $16,5 \times 24 \mathrm{~cm}, 349 \mathrm{p}$. (Bibliotheca di Storia delle religioni). ISBN : 88-86148-68-2.

Theos anaitios, «Dieu bors cause »: l'expression se rencontre à la lettre chez Platon, dans un unique passage des dialogues (Rép. X, 617e) où il est question du choix de vie qu'ont à faire les hommes avant leur réincarnation. Mais l'adjectif anaitios se retrouve, à trois reprises encore, appliquée à la divinité pour signifier que celle-ci n'est pas responsable des maux (Rép. II, 279b et c), ni de la malice dont se rendent coupables certains êtres (Tim., 42d, lois de la destinée des âmes édictées par le démiurge). Bien avant le disciple de Socrate, on rencontre, semble-t-il, l'expression daimon anaition dans un fragment mutilé d'Alcée (fr. 119 Voigt). Mais c'est dès Homère qu'apparaissent déjà les premiers germes d'une réflexion que la philosophie grecque se chargera de thématiser et que Leibniz, bien longtemps après, nommera "théodicée », entendant par là la justification d'une bonté divine que ne peut remettre en cause l'existence du mal dans le monde. Il est utile de préciser que le mot «teodicea» conserve en italien ce sens originel et particulier, alors que le mot français, tombé quelque peu en désuétude aujourd'hui, a vu sa signification s'élargir pour désigner la théologie qu'on appelle naturelle ou encore rationnelle, par opposition à la théologie dogmatique qui raisonne à l'intérieur d'une foi religieuse. La théodicée ne concerne donc qu'une partie des questions qui intéressent la théologie. Il reste que le problème de l'origine du mal et de la bonté divine, dans ses rapports avec les questions que 